\title{
Determinantal Systems of Apolar Triads in the Twisted Cubic.
}

\author{
By Dr W. L. Marr.
}

(Read and Received 19th June 1919.)

INTRODUCTION.

It has already been shown that nine points can be found on a conic to form six triads all apolar to a given triad $(A B C)$, the hessian lines of these six triads and of $A B C$ being concurrent.

The construction of the corresponding system in the twisted cubic is easy. In the first place, the poles of the six triads $P_{1} Q_{2} R_{3}$, etc., will lie on the plane of the triad $A B C$ to which they are all apolar. Secondly, the hessian lines of these triads, which in the conic were concurrent, will now be generating lines of a quadric circumscribing the twisted cubic. Now the pole of a triad is in the osculating plane at any of the points of the triad, hence the osculating plane at $P_{1}$ will contain the poles of the triads $P_{1} Q_{2} R_{3}$ and $P_{1} Q_{3} R_{2}$. But these poles are also the intersections of the hessian lines of $P_{1} Q_{2} R_{3}$ and $P_{1} Q_{3} R_{2}$ with the plane of $A B C$, and thus lie on the conic formed by the intersection of $A B C$ and the quadric just mentioned.

1. The nine point system for the twisted cubic is accordingly determined as follows:

Draw the osculating planes at $P_{1}, Q_{2}, R_{2}$ to cut the conic which is the intersection of the plane of the fundamental triad $A B C$ with the quadric which is generated by chords joining the corresponding points of the involution determined by the hessian points of $A B C$ and $P_{1} Q_{2} R_{3}$. The six points of intersection of these three osculating planes and the conic will be the six poles of the six triads, and from these six poles the nine points of the determinantal system can be readily found. 


\section{0}

2. Let the twisted cubic be given by

$$
x=p^{3}, y=3 p^{2}, z=3 p, t=1 \text {. }
$$

If the parameters of the hessian points of $P_{1} Q_{2} R_{3}$ be $h_{1}, h_{2}$, then $h_{1} h_{2}=K$ since the product of the parameters of the hessian points of any triad is constant.

The quadric generated by the six hessian lines of the six triads $P_{1} Q_{2} R_{3}$, etc., is

$$
3 z x-y^{2}-K\left(3 y t-z^{2}\right)=0 .
$$

The plane of $A B C$ (parameters $\left.-1,-\omega_{1}-\omega^{2}\right)$ is

$$
x+t=0 \text {. }
$$

The osculating plane at $P_{1}$ (parameter $l_{1}$ ) is

$$
x-l_{1} y+l_{1}^{2} z-l_{1}^{3} t=0 .
$$

The six poles of the six triads are the intersections of

and

$$
\begin{aligned}
& x\left(1+l^{3}\right)-y l+z l^{2}=0 \quad\left(\text { where } l=l_{1}, l_{2}, l_{3}\right) \\
& 3 z x-y^{2}+K\left(3 x y+z^{2}\right)=0 .
\end{aligned}
$$

3. Let the poles of $P_{1} Q_{2} R_{3}, P_{2} Q_{3} R_{1}$ and $P_{3} Q_{1} R_{2}$ be $U_{1}, U_{2}, U_{3}$ and of $P_{3} Q_{3} R_{2}, Q_{2} R_{1} P_{3}$ and $R_{3} P_{2} Q_{1}$ be $V_{1}, V_{2}, V_{3}$.

The line $U_{1} V_{1}$ is $x\left(1+l_{1}^{3}\right)-y l_{1}+z l_{1}^{2}=0$

$$
\begin{array}{ll}
U_{1} V_{2} \text { is } x\left(1+l_{2}^{3}\right) \text { etc. } & =0 \\
U_{1} V_{3} \text { is } x\left(1+l_{3}^{3}\right) \text { etc. } & =0
\end{array}
$$

for $U_{1} V_{1}$ is the intersection of the osculating plane at $P_{1}$ with $A B C$.

These lines cut $x=0$ when $y=z l_{1}, y=z l_{2}$, etc.

Hence the nine lines joining the vertices of $U_{1} U_{2} U_{3}$ to the vertices of $V_{1} V_{2} V_{3}$ cut the line $x=0$ in a determinantal system of points.

4. The line $x=0$ is the line joining the hessian poles of $U_{1} U_{2} U_{3}$ and $V_{1} V_{2} V_{3}$.

The equation of the conic may be written

or

$$
(y+K z)^{2}=(K y+z)\left(3 x \overline{1-K^{3}}+K \overline{K y+z}\right)
$$

where $Y=0$ is the line joining the pole $F$ of $A B C$ and the pole $M$ of the triangle which is apolar to $A B C$, and the parameters of whose hessian points are $\pm i \sqrt{ } K$. 
The line joining the hessian points of $P_{1} Q_{2} R_{3}$ meets the conic in $U_{1}$ whose coordinates are $x=K-\left(h_{1}+h_{2}\right)^{2}, y=3\left(K^{3}-\overline{h_{1}+h_{2}}\right)$, $z=3\left(K \overline{h_{1}+h_{2}}-1\right)$.

The parameter of $U_{1}$ with respect to $Y^{2}=Z X$ is $h_{1}+h_{2}$, and the parameters of $U_{1}, U_{2}, U_{3}$ are the roots of the cubic

the hessian line of which is

$$
p_{3}-3 K p+h_{1}^{3}+h_{2}^{3}=0
$$

$$
X-\frac{h_{1}^{3}+h_{2}^{3}}{K} Y+K Z=0,
$$

and hence the hessian poles of $U_{1} U_{2} U_{3}$ and $V_{1} V_{2} V_{3}$ lie on $X-K Z=0$, i.e. $x=0$.

This line $(x=0)$ is also the double tangent of the quartic (class 3) which is the envelope of $x\left(1+l^{3}\right)-y l+z l^{2}=0$.

5. The invariant $Q$ (v. Salmon's Higher Algebra) of the triads $U_{1} U_{2} U_{3}$ and $V_{1} V_{2} V_{3}$ vanishes.

This readily follows from the cubic equation for the parameters of $U_{1} U_{2} U_{3}$, and the corresponding equations for those of $V_{1} V_{2} V_{3}$, viz. $p^{3}-3 K p+k_{1}^{3}+k_{2}^{3}=0$.

It means that the pole of $x=0$ (the line joining their hessian poles) with respect to $U_{1} U_{2} U_{3}$ and $V_{1} V_{2} V_{3}$ is the same point $F$ on the conic which, as we have said, is also the pole of $A B C$ in the twisted cubic.

6. Relation to nodal cubic.

The coordinates of the line $U_{1} V_{1}$ are $1+l^{3},-l, l^{2}$.

Transforming from line to point let $x=1+l^{3}, y=-l, z=l^{2}$.

The quartic (class 3 ) which is the envelope of $U V$ now becomes a nodal cubic, viz. $y^{3}-z^{3}-x y z=0$.

i.e. $(x-3 y+3 z)\left(x-3 \omega y+3 \omega^{2} z\right)\left(x-3 \omega^{2} y+3 \omega z\right)=x^{3}$.

The conic which belongs to the four point family $A B C F$ now becomes a conic touching four lines which are the three tangents at the flexes and the line of the flexes of the nodal cubic.

Let $U_{1} V_{1}, U_{1} V_{2}, U_{1} V_{3}$ transform into three collinear points $X_{1}, X_{2}, X_{3}, U_{2} V_{1}, U_{2} V_{2}, U_{2} V_{3}$ into $Y_{1}, Y_{2}, Y_{3}$ and $U_{3} V_{1}$, etc., into $Z_{1}$, etc.

The rows and colums of

$$
\begin{array}{lll}
X_{1} & Y_{1} & Z_{1} \\
X_{2} & Y_{2} & Z_{2} \\
X_{3} & Y_{3} & Z_{3}
\end{array}
$$

are six tangents to the four-line conic. 
The theorem may be stated thus:-Given any conic touching the tangents at the three flexes and the line of flexes of a nodal cubic, then if tangents be drawn to the conic from three collinear points on the cubic, the line of which three points touches the conic, and tangents again be drawn to the conic from the points where the previous tangents cut the cubic, there will finally result a hexagon circumscribing the conic with nine of the intersections of the sides of the hexagon lying on the nodal cubic.

7. We have seen that if $U_{1} U_{2} U_{3}$ and $V_{1} V_{2} V_{3}$ be two triads on a conic for which the $Q$ invariant vanishes, the lines joining the vertices of one triad to the vertices of the other cut the line joining their hessian poles in a determinantal system of nine points.

Dr Milne has generalised this property to any pair of triads on a conic, $i e$. if $U_{1} U_{2} U_{3}$ and $V_{1} V_{2} V_{3}$ be any pair of triads, whether the $Q$ invariant vanishes or not, the lines $U_{1} V_{1}$, etc., cut the line joining their hessian poles in a determinantal system.

8. Conversely, if two triads are such that the joins of their vertices cut a line in a determinantal system the two triads are conconical, and the line is the line joining their hessian poles.

Let $U_{1} U_{2} U_{3}$ be a triad of points not collinear, and $P_{1} Q_{2} R_{3}$, etc., a nine point system on a straight line of which $H$ and $K$ are the hessian points of $A B C$, the triad collinear with $P_{1} Q_{2}$, etc., which is apolar to $\left(P_{1} Q_{2} R_{3}\right)$, etc.

The point $V_{1}$ will be the point of concurrence of $U_{1} P_{1}, U_{2} Q_{2}$, $U_{3} R_{3}$, and similarly for $V_{2}$ and $V_{3}$. We have to prove that $U_{1} U_{2} U_{3}$ and $V_{1} V_{2} V_{3}$ are con-conical.

Let $F, H, K$ be the triangle of reference, $F$ being the pole of $U K$ with regard to $U_{1} U_{2} U_{3}$.

$$
\begin{aligned}
& U_{2} U_{3} \equiv x+M_{1} y+N_{1} z \equiv X=0 \\
& U_{3} U_{1} \equiv x+M_{2} y+N_{2} z \equiv Y=0 \\
& U_{1} U_{2} \equiv x+M_{3} y+N_{3} z \equiv Z=0 .
\end{aligned}
$$

Since $F$ is the pole of $x=0$ (i e. $H K$ ) with respect to $U_{1} U_{3} U_{3}$ it follows that $x+M_{1} y+N_{1} z+x+M_{2} y+N_{2} z+x+M_{3},+N_{3} z$ is $x$. Therefore $M_{1}+M_{2}+M_{3}=0, N_{1}+N_{2}+N_{3}=0$.

Let the point $U_{1}$ be $\left(p_{1} q_{1} r_{1}\right)$

$$
\begin{aligned}
& U_{2} \text { be }\left(p_{2} q_{2} r_{2}\right) \\
& U_{3} \text { be }\left(p_{3} q_{3} r_{3}\right)
\end{aligned}
$$


Then $p_{1}=M_{2} N_{3}-M_{3} N_{2}=p_{2}=p_{3}$

$q_{1}=N_{2}-N_{3}, q_{2}=N_{3}-N_{1}$, etc.

$r_{1}=M_{3}-M_{2}, r_{2}=$ etc.

Let $V_{1}$ be $\left(x_{1} y_{1} z_{1}\right)$ so for $V_{2}$ and $V_{3}$.

The line $V_{1} U_{1}$ is

$$
x\left(q_{1} z_{1}-r_{1} y_{1}\right)+y\left(r_{1} x_{1}-p_{1} z_{1}\right)+z\left(p_{1} y_{1}-q_{1} x_{1}\right)=0 .
$$

$V_{1} U_{1}$ meets $x=0$ at the point $P_{1}$ which belongs to the determinantal system of collinear points, and has coordinates $\left(0 l_{1} 1\right)$.

Therefore $\frac{y}{z}=\frac{q_{1} x_{1}-p_{1} y_{1}}{r_{1} x_{1}-p_{1} z_{1}}=l_{1}$.

As $V_{1} U_{2}$ meets $x=0$ at $Q_{2}$ with coordinates $\left(0 l_{2} 1\right)$

$$
\frac{q_{2} x_{1}-p_{2} y_{1}}{r_{2} x_{1}-p_{2} z_{1}}=l_{2}
$$

and from $V_{1} U_{3} \frac{q_{3} x_{1}-p_{3} y_{1}}{r_{3} x_{1}-p_{3} z_{1}}=l_{3}$.

Hence

$$
\begin{aligned}
& x_{1}\left(q_{1}-r_{1} l_{1}\right)-p_{1} y_{1}+l_{1} p_{1} z_{1}=0 \\
& x_{1}\left(q_{2}-r_{2} l_{2}\right)-p_{2} y_{1}+l_{2} p_{2} z_{1}=0 \\
& x_{1}\left(q_{3}-r_{3} l_{3}\right)-p_{3} y_{1}+l_{3} p_{3} z_{1}=0 .
\end{aligned}
$$

The condition for concurrence of $U_{1} P_{1}, U_{3} Q_{2}, U_{3} R_{3}$ is found by eliminating $x y z$ from these three equations, and is

$$
\left(q_{1}-r_{1} l_{1}\right) p_{2} p_{3}\left(l_{2}-l_{3}\right)+\left(q_{2}-r_{2} l_{2}\right) p_{3} p_{1}\left(l_{3}-l_{1}\right)+\ldots=0,
$$

or since $p_{1}=p_{2}=p_{3}$ and $q_{1}=N_{2}-N_{3}$, etc.,

$$
\Sigma\left(N_{3}-N_{3}\right)\left(l_{3}-l_{3}\right)+\Sigma\left(M_{2}-M_{3}\right) l_{1}\left(l_{2}-l_{3}=0\right) \text {, }
$$

or

$$
N_{1} l_{1}+N_{2} l_{2}+N_{3} l_{3}=M_{1} l_{2} l_{3}+M_{2} l_{3} l_{1}+M_{3} l_{2} l_{2} \text {. }
$$

The line $V_{2} U_{2}$ will meet $x=0$ in $P_{3}$ with coordinates $\left(0 n_{1} 1\right)$.

The accompanying diagram will explain this.

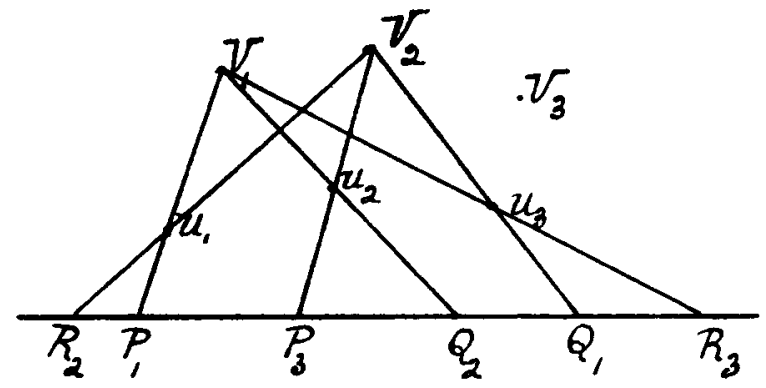


$U_{1} V_{1}, U_{1} V_{2}, U_{1} V_{3}$ give on $x=0 \quad P_{1} R_{2} Q_{3}$ corresponding to the $P_{1} Q_{2} R_{3}$ of $V_{1} U_{1}, V_{1} U_{2}, V_{1} U_{3}$.

Thus if $V_{2} U_{1}$ give $R_{2} V_{2} U_{2}$ must give $P_{3}$.

Where $V_{2} U_{2}$ meets $x=0$

$$
\frac{q_{2} x_{2}-p_{2} y_{2}}{r_{2} x_{2}-p_{2} z_{2}}=n_{1}=\frac{l_{1}\left(l_{2}-l_{3}\right)}{l_{1}-l_{2}}
$$

with corresponding equations resulting from $V_{2} U_{3}$ and $V_{2} U_{1}$.

The condition for concurrence is

$$
N_{1} l_{2}+N_{2} l_{3}+N_{3} l_{1}=M_{2} l_{2} l_{3}+M_{3} l_{3} l_{1}+M_{1} l_{1} l_{2} .
$$

The condition for the concurrence of $V_{3} U_{3}, V_{3} U_{1}, V_{3} U_{2}$ is in the same way $N_{1} l_{3}+\ldots=M_{1} l_{3} l_{1}+\ldots$

The following three consistent equations must be satisfied :

Square and add

$$
\begin{aligned}
& N_{1} l_{1}+\ldots=M_{1} l_{2} l_{3}+\ldots \\
& N_{1} l_{2}+\ldots=M_{1} l_{1} l_{2}+\ldots \\
& N_{1} l_{3}+\ldots=M_{1} l_{3} l_{2}+\ldots
\end{aligned}
$$

$$
\begin{aligned}
& \left(N_{1}^{2}+N_{2}^{2}+N_{3}^{2}\right)\left(l_{1}^{2}+l_{2}^{2}+l_{3}^{2}\right)+2\left(N_{2} N_{3}+\ldots\right)\left(l_{2} l_{3}+\ldots\right) \\
& =\left(M_{1}^{2}+M_{2}^{2}+M_{3}^{2}\right)\left(l_{2}^{2} l_{3}^{2}+\ldots\right)+2\left(M_{2} M_{3}+\ldots\right)\left(l_{1}^{2} l_{2} l_{3}+\ldots\right)
\end{aligned}
$$

But $\Sigma 2 N_{2} N_{3}=-\Sigma N_{1}^{2}$ and $\Sigma 2 M_{2} M_{3}=-\Sigma M_{1}^{2}$.

Therefore

$$
\begin{aligned}
& \left(N_{1}^{2}+N_{2}^{2}+N_{3}^{2}\right) \Sigma\left(l_{2}-l_{3}\right)^{2}=\left(M_{1}^{2}+M_{2}^{3}+M_{3}^{2}\right) \Sigma l_{1}^{2}\left(l_{1}-l_{3}\right)^{2} . \\
& \text { But } \quad \frac{\sum l_{1}^{2}\left(l_{2}-l_{3}\right)^{2}}{\Sigma\left(l_{2}-l_{3}\right)^{2}}=K
\end{aligned}
$$

for the term on the left side is the product of the parameters of the hessian points of any triad in the determinantal system, and is constant $(K)$. Hence $N_{1}^{2}+N_{2}^{2}+N_{3}^{2}=K\left(M_{1}^{2}+M_{2}^{2}+M \frac{2}{3}\right)$.

To find the locus of $V_{1} V_{2} V_{3}$ we have only to make use of the relation $\Sigma l_{1}^{2}\left(l_{2}-l_{3}\right)^{2}=K \Sigma\left(l_{2}-l_{3}\right)^{2}$, substituting for $l_{1} l_{2} l_{3}$ the expressions in $x, y, z, p, q, r$ already found.

The locus of $V$ thus is

$$
\begin{gathered}
\sum\left(\frac{q_{1} x-p_{1} y}{r_{1} x-p_{1} z}\right)^{2}\left(\frac{q_{2} x-p_{2} y}{r_{2} x-p_{2} z}-\frac{q_{3} x-p_{3} y}{r_{3} x-p_{3} z}\right)^{2} \\
=K \Sigma\left(\frac{q_{2} x-p_{2} y}{r_{2} x-p_{2} z}-\frac{q_{3} x-p_{3} y}{r_{3} x-p_{3} z}\right)^{2}
\end{gathered}
$$

or $\Sigma\left(x+M_{1} y+N_{1} z\right)^{2}\left[\left\{\left(N_{2}-N_{3}\right) x-y\right\}^{2}-K\left\{\left(M_{3}-M_{2}\right) x-z\right\}^{2}\right]=0$.

This quartic passes through $\mathbb{F}(100)$ since

$$
\Sigma\left(N_{2}-N_{3}\right)^{2}=K \Sigma\left(M_{3}-M_{2}\right)^{2}
$$


On transferring to $U_{1} U_{2} U_{3}$ as triangle of reference the quartic becomes

$$
\Sigma X^{2}\left[\left(N_{3} Y-N_{2} Z\right)^{2}-K\left(M_{3} Y-M_{2} Z\right)^{2}\right]=0 .
$$

Use $\Sigma\left(N_{1}^{2}-K M_{1}^{2}\right)=0$ and the quartic breaks up into the two conics and

$$
\begin{gathered}
Y Z\left(N_{1}^{2}-K M_{1}^{2}\right)+Z X\left(N_{2}^{2}-K M_{2}^{2}\right)+X Y\left(N_{3}^{2}-K M_{3}^{2}\right)=0, \\
Y Z+Z X+X Y=0 .
\end{gathered}
$$

The first conic which alone is related to the nine points passes through $F^{\prime}(111)$, and therefore the hessian pole of $U_{1} U_{2} U_{3}$ lies on $X+Y+Z=0$ or $x=0$. Hence if two triads by the joining of their vertices give a determinantal system on a line these triads must be con-conical, and the line must contain their hessian poles.

9. The planes $P_{1} P_{2} P_{3}, Q_{1} Q_{2} Q_{3}, R_{1} R_{2} R_{3}$ in the twisted cubic intersect in a line on the plane $A B C$ and $P_{1} Q_{1} R_{1}$, etc., in another line also on $A B C$. These lines are $K x+y=0$ and $K z-t=0$, and can be interpreted in terms of the central triad (paragraph 4), the pole of which is $M$ and the parameters of its hessian points $\pm i \sqrt{ } K$. 